\title{
The peritoneal macrophage inflammatory profile in cirrhosis depends on the alcoholic or hepatitis C viral etiology and is related to ERK phosphorylation
}

Ana Tapia-Abellán ${ }^{1+}$, María Martínez-Esparza ${ }^{1{ }^{*}+}$, Antonio J Ruiz-Alcaraz ${ }^{1}$, Trinidad Hernández-Caselles ${ }^{1}$, Cristina Martínez-Pascual ${ }^{2}$, Manuel Miras-López², José Such ${ }^{3,4}$, Rubén Francés ${ }^{3,4}$ and Pilar García-Peñarrubia ${ }^{1}$

\begin{abstract}
Background: The development of ascites in cirrhotic patients generally heralds a deterioration in their clinical status. A differential gene expression profile between alcohol- and hepatitis C virus (HCV)-related cirrhosis has been described from liver biopsies, especially those associated with innate immune responses. The aim of this work was to identify functional differences in the inflammatory profile of monocyte-derived macrophages from ascites in cirrhotic patients of different etiologies in an attempt to extrapolate studies from liver biopsies to immune cells in ascites. To this end 45 patients with cirrhosis and non-infected ascites, distributed according to disease etiology, HCV $(n=15)$ or alcohol $(n=30)$ were studied. Cytokines and the cell content in ascites were assessed by ELISA and flow cytometry, respectively. Cytokines and ERK phosphorylation in peritoneal monocyte-derived macrophages isolated and stimulated in vitro were also determined.
\end{abstract}

Results: A different pattern of leukocyte migration to the peritoneal cavity and differences in the primed status of macrophages in cirrhosis were observed depending on the viral or alcoholic etiology. Whereas no differences in peripheral blood cell subpopulations could be observed, T lymphocyte, monocyte and polymorphonuclear cell populations in ascites were more abundant in the HCV than the alcohol etiology. HCV-related cirrhosis etiology was associated with a decreased inflammatory profile in ascites compared with the alcoholic etiology. Higher levels of IL-10 and lower levels of IL-6 and IL-12 were observed in ascitic fluid from the HCV group. Isolated peritoneal monocyte-derived macrophages maintained their primed status in vitro throughout the $24 \mathrm{~h}$ culture period. The level of ERK1/2 phosphorylation was higher in ALC peritoneal macrophages at baseline than in HCV patients, although the addition of LPS induced a greater increase in ERK1/2 phosphorylation in HCV than in ALC patients.

Conclusions: The macrophage inflammatory status is higher in ascites of alcohol-related cirrhotic patients than in HCV-related patients, which could be related with differences in bacterial translocation episodes or regulatory T cell populations. These findings should contribute to identifying potential prognostic and/or therapeutic targets for chronic liver diseases of different etiology.

Keywords: Ascites, Cirrhosis, Cytokines, Etiology, MAP kinases, HCV, Alcohol

\footnotetext{
*Correspondence: maria@um.es

${ }^{\dagger}$ Equal contributors

'Departamento de Bioquímica, Biología Molecular (B) e Inmunología

Facultad de Medicina, Universidad de Murcia, Murcia 30100, Spain

Full list of author information is available at the end of the article
} 


\section{Background}

Alcoholic liver disease (ALD) and hepatitis C virus (HCV) infection are the two major causes of chronic liver diseases in the developed world [1]. The development of ascites, an accumulation of fluid in the peritoneal cavity, in a cirrhotic patient generally runs parallel with deterioration in clinical status and presages a poor prognosis. While the overall cirrhosis mortality rate has falling in the last three decades [2], $\mathrm{HCV}$ mortality rates, closely associated with cirrhosis, have been increasing since the 1990s [3]. For the clinical management of cirrhosis, it is important to assess whether the differences in the outcomes of cirrhosis depend on the etiology. In this respect, the histopathology of alcohol-induced cirrhosis (ALC-C) and HCV-induced cirrhosis (HCV-C) is very similar and characteristic fibrosis patterns leading to cirrhosis can overlap in them [4]. Nonetheless, little is known regarding the differences and/or similarities between alcohol- and HCV- induced liver disease at the molecular level [5] and, to the best of our knowledge, no data on human peritoneal cells have bee reported.

Global transcriptional profiling using oligonucleotide microarrays on liver biopsies from patients with cirrhosis showed that some genes are differentially expressed between ALC-C and HCV-C. Many of the gene expression changes specifically observed in $\mathrm{HCV}$-induced cirrhotic livers were related to activation of the innate antiviral immune response, while differential mechanisms between chronic liver damage due to $\mathrm{HCV}$ or ethanol may be related to regulation of the lipid metabolism and macrophage activation resulting in the deposition of extracellular matrix components $[1,5]$. Additionally, several studies have demonstrated that alcohol exposure activates innate immunity and induces several pro-inflammatory cytokines, including TNF- $\alpha$, subsequently inducing hepatocellular damage. The activation of innate immunity also results in higher levels of hepatoprotective intermediaries, such as IL6 , and anti-inflammatory cytokines, such as IL-10, which play an important role in ameliorating alcoholic liver injury and inflammation [6]. However, chronic alcohol exposure attenuates the signaling pathways triggered by these cytokines, thereby limiting their anti-inflammatory and hepatoprotective effects, and contributing to the development of ALD [7].

Monocyte-derived macrophages (M-DM) play an important role as antigen-presenting cells of the innate immune response established against pathogen associated molecular patterns (PAMPs), including bactDNA and LPS [8]. In this respect, we reported bactDNA-activated cell-mediated immune response and nitric oxide overproduction through the inducible form of NO synthase in peritoneal macrophages from patients with cirrhosis and ascites [9] and, more recently, the primed status of peritoneal M-DM from cirrhotic patients related to ERK phosphorylation and IL-6 secretion [10].
This study focuses on the inflammatory profile of ascites and peritoneal M-DM from patients with ALC-C or $\mathrm{HCV}-\mathrm{C}$ to assess whether the immune status and inflammatory mechanisms leading to end-stage liver cirrhosis present a different pattern, depending on the agent that caused this pathology, and extrapolate out from previous studies performed in liver biopsies to immune cells in ascites.

\section{Results}

\section{Patient baseline characteristics}

A consecutive series of 61 patients with cirrhosis and ascites was initially recruited for the study. Sixteen patients were excluded due to episodes of gastrointestinal bleeding in the previous 2 weeks $(\mathrm{n}=2)$, culture-positive ascites $(n=3)$, hepatocellular carcinoma $(n=2)$, mixed alcoholic and viral etiology $(n=4)$, hepatitis $B$ virus infection etiology $(n=1)$ or continuous use of norfloxacin as secondary prophylaxis of spontaneous bacterial peritonitis $(\mathrm{n}=4)$. Finally 45 patients fulfilling all the inclusion criteria were included in the study, distributed according to disease etiology, HCV-C $(n=15)$ or ALC-C $(n=30)$. The clinical and analytical characteristics of patients are detailed in Table 1. Blood and ascites cultures were negative in all cases. None of the patients died during hospitalization or developed spontaneous bacterial peritonitis.

As can be observed from Table 1, the absolute total number of ascitic leukocytes found in patients with HCV-C was significantly higher than in ALC-C patients. These differences were not detected in blood.

There were no significant differences as regards the rest of the clinical and analytical characteristics between the two groups of patients studied.

During 6-month follow-up study, three patients from the ALC-C group and one patient from the HCV-C group died. The causes of death in the ALC-C group were liver insufficiency $(n=2)$ and renal failure $(n=1)$. In the HCV-C group, the cause of death was renal failure $(n=1)$.

\section{$\mathrm{HCV}-\mathrm{C}$ is associated with increased counts of some leukocyte subpopulations in ascites}

To further explore differences in the number of leukocytes present in the ascites of both groups, the leukocyte subpopulations were characterized by flow cytometry analysis based on both the morphology and $C D$ receptor expression criteria. The results presented in Table 2 show that $\mathrm{T}$ lymphocytes, PMNs and M-DM counts were high in the ascites of $\mathrm{HCV}-\mathrm{C}$ patients compared with the ALC-C group. A study of subset cell type distribution in blood showed no significant differences between ALC-C and HCV-C patients (data not shown). 


\begin{tabular}{|c|c|c|}
\hline \multirow{2}{*}{$\begin{array}{ll} & \text { Variable } \\
\text { Etiology } & \\
\end{array}$} & \multicolumn{2}{|c|}{$\begin{array}{l}\text { Patients with cirrhosis and } \\
\text { culture-negative ascites }(n=45)\end{array}$} \\
\hline & ALC-C (30) & HCV-C (15) \\
\hline Age & $58.5(12.5)$ & $58(24)$ \\
\hline Male sex n (\%) & $30(100.0)$ & $14(93.3)$ \\
\hline Previous episodes of ascites n (\%) & $25(83.3)$ & 13 (86.6) \\
\hline Child-Pugh mean score & $10(3)$ & $9(3)$ \\
\hline Meld mean score & $14.5(10)$ & $14(5)$ \\
\hline Bilirubin (mg/dl) & $2.7(3)$ & $1.85(1.7)$ \\
\hline Albumin (g/dl) & $2.9(1.1)$ & $2.85(0.9)$ \\
\hline Quick (\%) & $64(18)$ & $54(24)$ \\
\hline Serum creatinine (mg/dl) & $1.07(1.2)$ & $1(0.5)$ \\
\hline Serum sodium (mEq/l) & $134(6.3)$ & $136(7)$ \\
\hline INR & $1.45(0.3)$ & $1.46(0.6)$ \\
\hline Blood WBC/mm $\mathrm{mm}^{3}$ & $4260(2400)$ & $3930(4040)$ \\
\hline Ascites $\mathrm{WBC} / \mathrm{mm}^{3}$ & $21.29(47.4)$ & $49(82.8)^{*}$ \\
\hline Ascites Total protein (g/dl) & $1.7(0.3)$ & $1.6(1)$ \\
\hline
\end{tabular}

Continuous variables are expressed as median (interquartile range) and categorical variables as percentage. WBC: white blood cells; Mann-Whitney U test ${ }^{*} P<0.05$.

Anti-inflammatory cytokine levels are higher and proinflammatory levels are lower in the ascites of HCV-C cirrhotic patients

Figure 1 shows the concentration in $\mathrm{pg} / \mathrm{ml}$ of IL-10, IL12 , IL-1 $\beta$, IL- 6 and TNF- $\alpha$. The results showed that the ascites from the HCV-C group had lower levels of proinflammatory cytokines, although the differences between both groups were statistically significant only in the case of IL-12. The results also revealed that IL-10 anti-inflammatory cytokine levels in the ascites of HCV$\mathrm{C}$ patients were significantly higher than the corresponding levels from the ALC-C group of cirrhotic patients.

\section{Peritoneal M-DM isolated from ascites of cirrhotic} patients maintain their cytokine secretory profile in vitro We next compared the cytokine production ability of the peritoneal M-DM from $\mathrm{HCV}-\mathrm{C}$ and $\mathrm{ALC}-\mathrm{C}$ patients,

Table 2 White blood cell distribution in ascites

\begin{tabular}{lll}
\hline & \multicolumn{2}{c}{ Etiology } \\
\cline { 2 - 3 } Cell type $/ \mathbf{m m}^{3}$ & ALC-C & HCV-C \\
\hline PMNs & $0.5(4.4)$ & $3.4(7)^{*}$ \\
M-DM & $6.95(12.6)$ & $14.8(19.9)^{*}$ \\
T Lymphocytes & $7.37(12.6)$ & $14,66(22.6)^{*}$ \\
B Lymphocytes & $0.16(0.5)$ & $0.21(0.3)$ \\
NK cells & $1.22(2.2)$ & $2.01(5.6)$ \\
\hline
\end{tabular}

Results are expressed as median (interquartile range). Mann-Whitney $U$ test: ${ }^{*} P<0.05$. first exploring the relative contribution of the M-DM to the cytokines contained in ascites by referring the concentration of cytokines to the number of this cell type (Figure 2A). Then we analyzed the basal production of cytokines in $24 \mathrm{~h}$ cultures of the M-DM population isolated from ascites (Figure 2B).

The results revealed that $\mathrm{M}-\mathrm{DM}$ from the $\mathrm{HCV}-\mathrm{C}$ group produces significantly lower levels of IL-12, but also of IL-6 and TNF- $\alpha$ cytokines, than the ALC-C group when M-DM were isolated and cultured in vitro (Figure 2B). These data are consistent with those of cytokine levels in the ascites (Figure 2A), indicating that M-DM are "primed" by the pathological microenvironment and preserve their secretory profile, being more pro-inflammatory in the case of ALC-C group.

No significant differences in IL-10 production were observed between the two groups as regards a M-DM cultured in vitro, nor with respect to M-DM present in ascites, indicating that M-DM are not the main cell type responsible for the differences found for this cytokine in ascites.

\section{Peritoneal M-DM isolated from ascites of cirrhotic} patients are able to further respond to in vitro stimulation with several microbial stimuli

Spontaneous bacterial peritonitis (SBP) caused by the translocation of intestinal bacteria and their products, such as LPS or bacterial DNA, is one of the main lifethreatening complications of cirrhosis. Given this, the quality and intensity of the peritoneal immune response plays a crucial role in the prognosis of these compromised patients. To seek further insight into the ability of cirrhotic peritoneal M-DM to respond to different PAMPs, we studied the capability of isolated M-DM to respond to LPS, synthetic CpG-ODNs and heat-killed $C$. albicans as examples of stimuli able to trigger activation and phagocytosis through different PRRs. For this purpose, the cytokines produced by M-DM cultured for $24 \mathrm{~h}$ in the presence of these stimuli were studied. The results shown in Table 3 reveal that this cell population is able to further recognize and respond to different PAMPs, although significant differences were observed, depending on the cytokine in question and the specific stimuli applied. The results shown in Table 3 point to a, first strong significant response of TNF- $\alpha$ secretion in the presence of LPS and C. albicans in M-DM from both groups of patients. Second, IL-6 and IL-10 secretion was significantly increased in all stimulatory conditions, except in the M-DM from ALC-C patients after stimulation by ODN. Third, the relative increases in IL-6 was significantly higher in $\mathrm{HCV}-\mathrm{C}$ for the three stimuli assayed, while the increase in IL-10 secretion was only significant in the ALC-C group stimulated by ODN. Fourth, secreted IL-12 was only significantly increased 


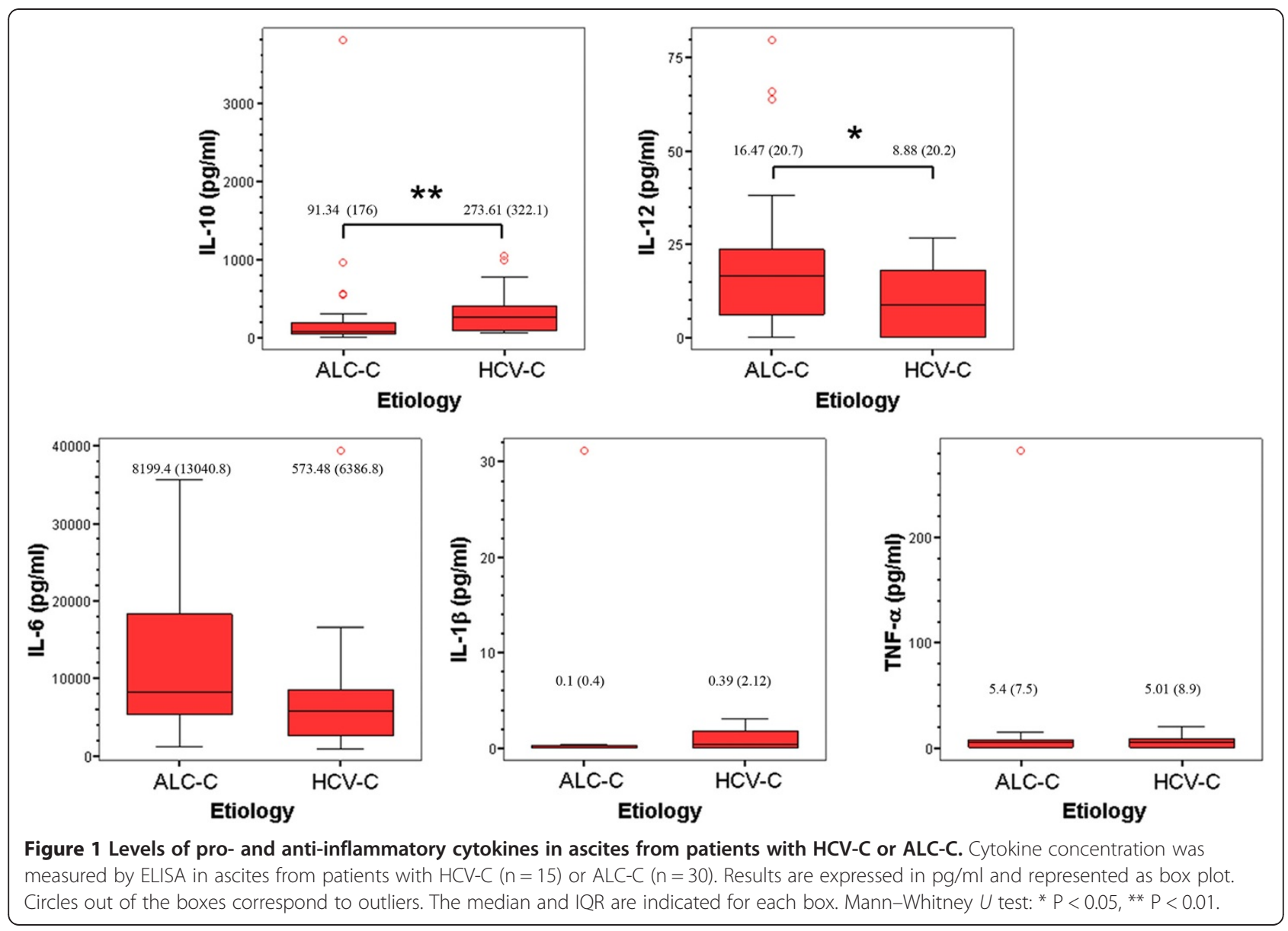

by LPS and C. albicans in M-DM obtained from ALC-C patients. Finally, LPS and C. albicans induced a significant increase in secreted IL-1 $\beta$ in M-DM from both groups of patients compared with their respective controls, although no significant differences were observed between groups.

\section{ERK1/2 phosphorylation levels are lower in peritoneal M-} DM isolated from ascites of patients with HCV-C vs. ALC-C We recently described the primed status of peritoneal macrophages in cirrhosis, finding it to be related to ERK1/2 phosphorylation and IL-6 secretion [10]. We therefore further analyzed the phosphorylation of ERK1/ 2 at baseline and in the presence of LPS in M-DM from both group of patients. As shown in Figure 3, the results confirmed the primed status of ERK1/2 phosphorylation in peritoneal M-DM from ALC-C patients, which is consistent with the high concentration of IL- 6 with respect to M-DM in ALC-C ascites, as well as with the IL-6 level produced in vitro by isolated M-DM cells from ALC-C patients. In contrast, baseline phosphorylation of ERK1/2 in M-DM from HVC-C patients was very low, although its relative increase after exposure to LPS for
15 min was higher than the corresponding increase in M-DM from ALC-C group. Again, this observation is consistent with the relative increase of IL- 6 after LPS stimulation described above. These results confirm and extend the positive correlation previously established between basal IL-6 levels and ERK phosphorylation in peritoneal M-DM from patients with cirrhosis [10], and point to significant differences between ALC-C and $\mathrm{HCV}-\mathrm{C}$ patients as regards the primed status at baseline.

\section{Discussion}

Clinical research in human diseases is largely based on samples obtained by surgical or aggressive procedures from extremely ill patients. This implies the use of convenience samples of limited size rather than larger samples drawn at random from the population. Therefore, the main limitation of this type of study on the immune status in cirrhotic ascites is the nature and size of the sample. To minimize this limitation we processed consecutive samples obtained from cirrhotic patients from two different institutions during a three year period. A robust statistical test was used to ensure that the observed differences were significant. Nevertheless, this 


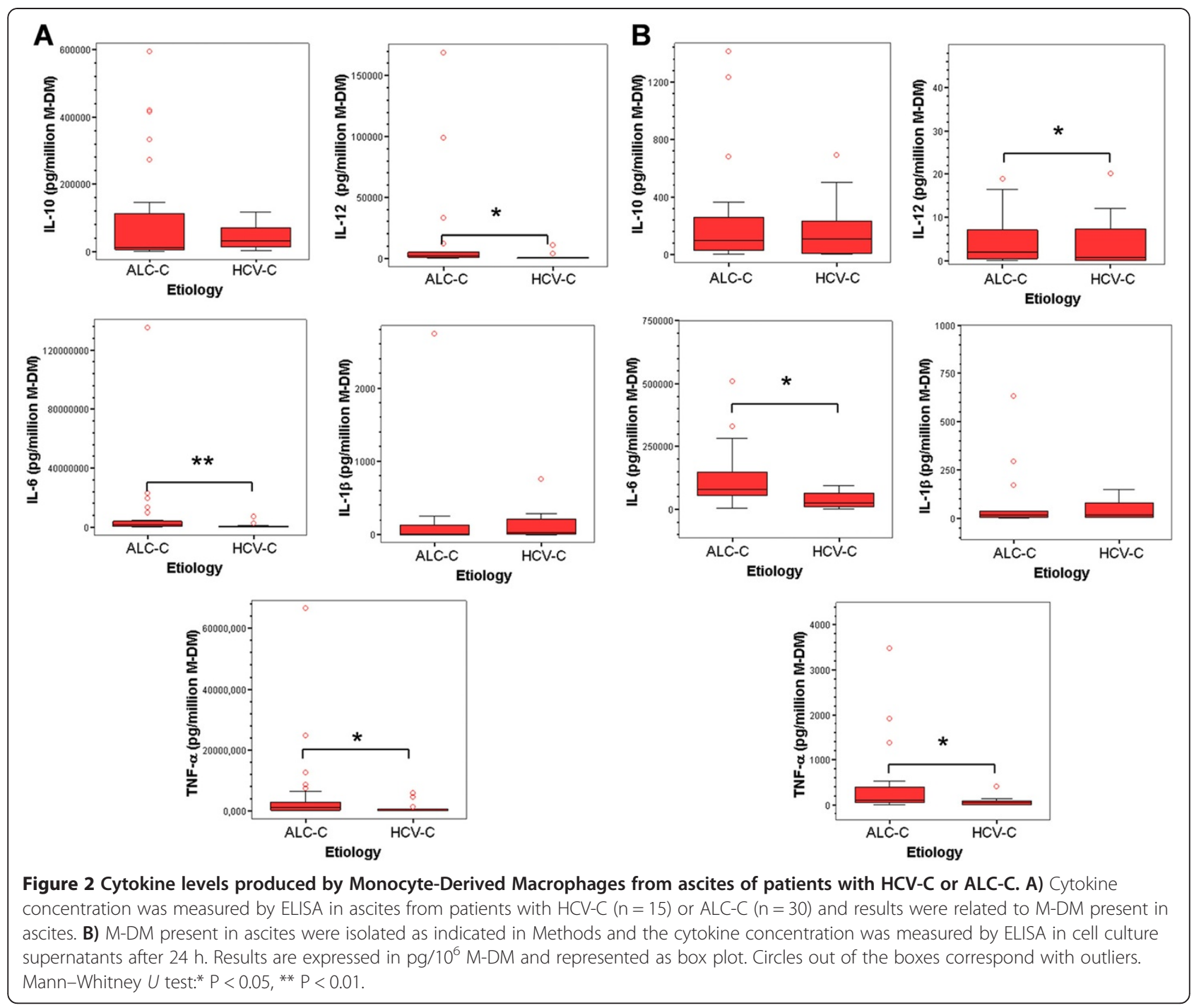

Table 3 Monocyte-Derived Macrophages response to in vitro stimulation

\begin{tabular}{|c|c|c|c|c|}
\hline \multirow[t]{2}{*}{ Cytokines } & \multirow[t]{2}{*}{ Etiology } & \multicolumn{3}{|c|}{ Fold activation } \\
\hline & & LPS & ODN & C. albicans \\
\hline \multirow[t]{2}{*}{ TNF-a } & ALC-C & $89.26(204.5)^{a}$ & $0.89(1.7)$ & $170.92(374.6)^{a}$ \\
\hline & HCV-C & $97.61(163.9)^{a}$ & $2.42(4.9)$ & $4334.96(595.4)^{a}$ \\
\hline \multirow[t]{2}{*}{ IL-6 } & ALC-C & $2.73(3.5)^{\mathbf{a}}$ & $0.84(5.8)$ & $3.35(10.4)^{\mathbf{a}}$ \\
\hline & HCV-C & $5.97(19.6) *$ *a & $1.26(7.2)^{*, \mathbf{a}}$ & $5.68(37.2) *$ *a \\
\hline \multirow[t]{2}{*}{ IL-10 } & ALC-C & $9.06(6.2)^{\mathbf{a}}$ & $0.77(0.6)$ & $13.7(24.5)^{\mathbf{a}}$ \\
\hline & HCV-C & $11.33(13.9)^{\mathrm{a}}$ & $1.4(2.6) *, \mathrm{a}$ & $223.4(45.3)^{\mathbf{a}}$ \\
\hline \multirow[t]{2}{*}{ IL-12 } & ALC-C & $1.75(3.4)^{\mathbf{a}}$ & $2.1(8.4)$ & $3.7(6.6)^{a}$ * \\
\hline & HCV-C & $3.15(4.8)$ & $6.6(17.4)$ & $1.93(1.1)$ \\
\hline \multirow[t]{2}{*}{ IL-1 $\beta$} & ALC-C & $20.21(63)^{a}$ & $1.5(10)$ & $46.85(135.6)^{a}$ \\
\hline & HCV-C & $10.89(24.7)^{\mathbf{a}}$ & $2.11(2.73)$ & $15.4(28.5)^{\mathbf{a}}$ \\
\hline
\end{tabular}

Results are expressed as median (interquartile range). Mann-Whitney $U$ test: ${ }^{*} P<0.05$ between ALC-C and HCV-C; Wilcoxon signed-rank test: ${ }^{a} P<0.05$ between control and treatments. still does not solve the important question of whether results observed in samples of convenience can be generalized to the larger population. Herein we show first, that compared with ALC-C, HCV-C associated ascites contains a significantly higher number of leukocytes, although these findings did not match the cellular distributions observed in the peripheral blood. This indicates that the absolute cell number and population distribution of peritoneal leukocytes in cirrhotic ascites varies with the underlying cause and does not mirror the situation in peripheral blood. In this regard it is important to remark that the majority of studies on immune cells in human cirrhosis have been performed in blood samples, mainly due to the difficulty involved in obtaining and managing ascites $[11,12]$ and in to a lesser extent, in liver biopsies $[13,14]$. Thus, it is interesting to further explore the ascites in order to extrapolate data on immune cells from the above three types of samples to better understand the mechanisms underlying the 


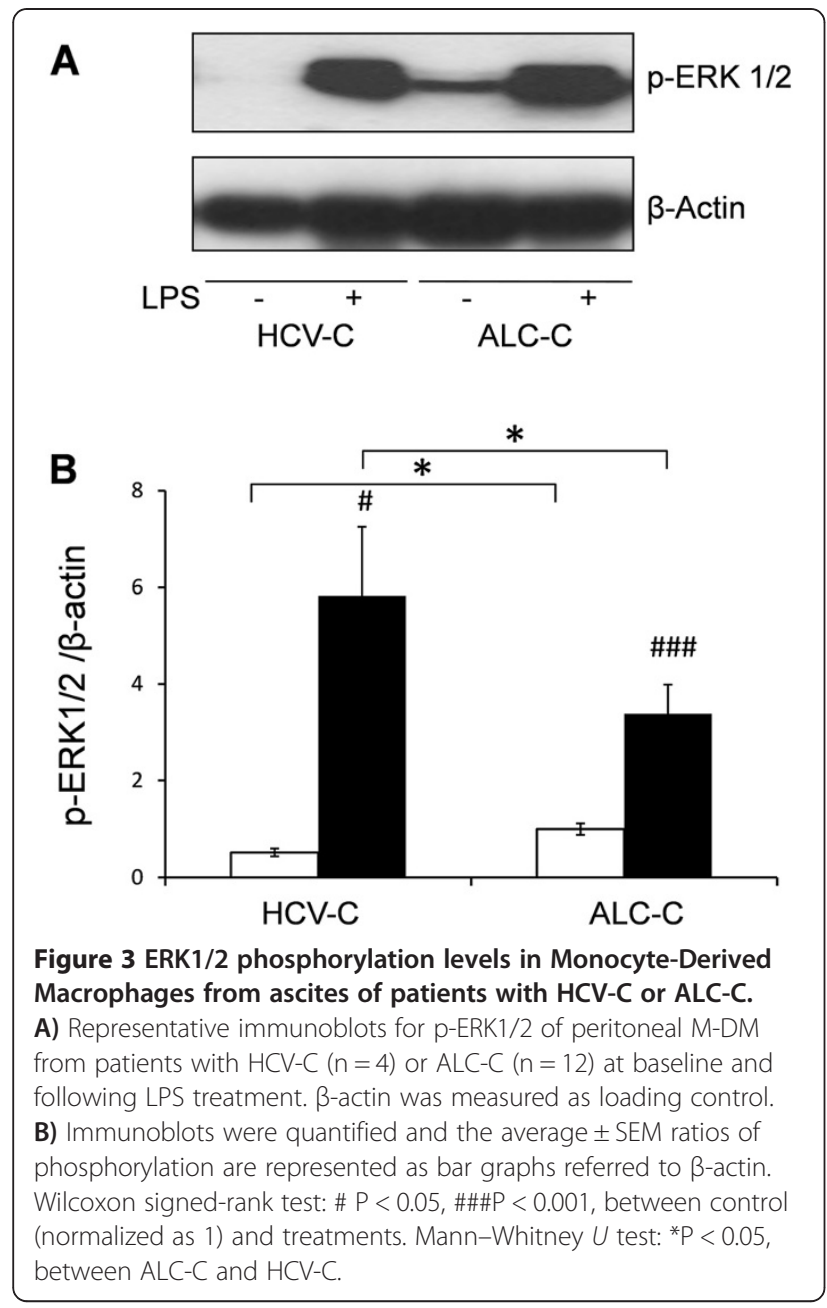

pathogenesis of liver damage, enabling doctors to predict the risks and outcomes of apply the appropriate treatments.

Furthermore the findings also reveal that leukocyte migration towards the peritoneal cavity is not a passive process induced by hemodynamic anomalies associated to cirrhosis, like portal hypertension, but more of an active chemoattractant-induced process, recruiting leukocytes not only from blood vessels, but also from impaired lymphatic drainage [15]. Moreover the higher number of leukocytes in ascites from $\mathrm{HCV}-\mathrm{C} v s$. ALC-C group resulted from increases in the $\mathrm{T}$ lymphocyte, PMN and monocyte cell subpopulations. This suggests that a specific differential pattern of chemoattractant stimuli must be involved in the recruitment of each particular cell population, depending on the microbial stimuli involved in the cirrhosis etiology. In this regard, it has been shown that the expression of CCR1, $-2,-5$ and $\mathrm{CXC}-1$ is induced in patients with chronic liver diseases, so that intrahepatic increases of CCL3-5 and IL-8 chemokines would be involved in recruiting not only monocyte/macrophages but also other immune cell populations $[16,17]$.

Our results also showed that ascites from $\mathrm{HCV}-\mathrm{C}$ patients exhibits a significantly lower concentration of IL-12 pro-inflammatory cytokine and a significant higher amount of the anti-inflammatory cytokine IL-10 compared with the ALC-C group, suggesting a predominant Th2/Treg profile in the pathogenesis of advanced HCVC. Indeed, it has been described that $\mathrm{HCV}$ by itself induces intrahepatic Tr1 cells [18], expands peripheral Treg in patients with normal aminotransferases [11] and affects dendritic cell function, switching the cytokine profile towards a suppressive phenotype of IL-10 and transforming growth factor beta predominance, preventing cell maturation and inhibiting the allostimulating capacity [19]. However, when cytokine levels were compared with the number of M-DM contained in ascites of these patients, or secreted in vitro by isolated peritoneal M-DM, apart from differences in IL-12, the concentration of IL- 6 and TNF- $\alpha$ also significantly differed between both groups of patients. Furthermore, this fact was associated with lower baseline ERK1/2 phosphorylation than observed in the ALC-C group. Four conclusions can be drawn from these findings. First, these results confirm and extend the positive correlation established between basal IL-6 levels and ERK phosphorylation in peritoneal M-DM from patients with cirrhosis [10]. Second, they indicate that M-DM are "differently primed" by the in vivo pathophysiological environment and preserve their inflammatory differentiation profile for at least $24 \mathrm{~h}$, being more pro-inflammatory in the case of ALC-C. This "alert state" could provide an advantage for preventing the development of SBP in intermittent events of intestinal bacterial translocation in ALC-C patients. Third, they confirm the predominantly immune inhibited status of M-DM in the end-stages of $\mathrm{HCV}$-induced hepatic damage compared with ALC-C [20], which may be intended to prevent immunemediated decompensation. Fourth, the drop of significant differences in IL-10 levels with respect to M-DM strongly suggests that other immune cells are contributing to the total amount of this anti-inflammatory cytokine in ascites of the $\mathrm{HCV}-\mathrm{C}$ group, especially regulatory Th subpopulations. However, this does not mean that $\mathrm{M}-\mathrm{DM}$ from $\mathrm{HCV}-\mathrm{C}$ are functionally exhausted or endotoxin-tolerant as described [21], since they are able to further respond to stimulation by several PAMP agonists. In fact, the relative increase of IL-6 secretion and ERK1/2 phosphorylation in isolated M-DM stimulated by LPS was significantly higher in $\mathrm{HCV}-\mathrm{C}$ than in ALC$\mathrm{C}$ patients. The same results were obtained for IL-6 secretion induced by ODN and C. albicans, and IL-10 induced by ODN from the $\mathrm{HCV}-\mathrm{C}$ group. The above 
findings also point to the differential expression and/or susceptibility to ligands of PRRs in the M-DM present in ascites from both types of cirrhotic patient. Supporting this hypothesis, the clear up-regulation of TLRs has been reported in specimens from patients with HCV infection $[22,23]$ and ALC-induced liver damage (reviewed in [24]).

SBP caused by translocation of intestinal bacteria and their products such as LPS or bacterial DNA is one of the main life-threatening complications of cirrhosis [25]. Faced with this condition, the quality and intensity of the peritoneal immune response seemingly plays a crucial role in the prognosis of these compromised patients. The lower inflammatory profile of ascites of the HCV-C compared with alcoholic etiology could also be the result of the hypothetically lower frequency of intestinal bacterial translocation in $\mathrm{HCV}-\mathrm{C}$ patients. Our results indicate the need for additional studies with a larger clinical sample to answer the question as to whether or not the difference in the MD-M peritoneal inflammatory status could influence the clinical outcome of patients beyond six months or the tolerance of a transplanted liver.

\section{Conclusions}

This study shows for the first time, that the concentration of T lymphocyte, PMN and monocyte subpopulations in ascites of patients with HCV-C is higher than that of patients with ALC-C, while no significant differences exist in peripheral blood. This indicates the lack of correlation between circulating white blood cell numbers and peritoneal leukocytes in cirrhotic ascites. The study provides important information on some differential characteristics of cirrhotic peritoneal M-DM that will allow extrapolation to previous data obtained in peripheral blood and liver biopsies. This will contribute to a better understanding of the mechanisms underlying the pathogenesis of liver damage leading to decompensated cirrhosis of differing etiologies. Thus, HCV etiology is associated with a predominant immune inhibitory status in ascites and isolated peritoneal M-DM unlike the cirrhosis induced by alcohol. A better knowledge of the cellular and molecular pathways of M-DM activation in cirrhosis could contribute to the design of further studies to identify potential prognostic and/or therapeutic targets for liver fibrosis of different etiologies.

\section{Methods}

\section{Patients}

Patients were admitted at the Liver Unit of Hospital General Universitario, Alicante, Spain, or at the Liver Transplant Unit of Hospital Universitario Virgen de la Arrixaca, Murcia, Spain. Cirrhosis was diagnosed by histology or by clinical, laboratory, and/or ultrasonographic findings. Exclusion criteria were the presence of a culture-positive blood or ascites, an ascites poly-

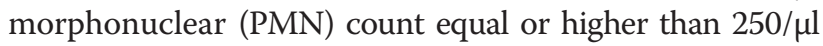
[26], signs or symptoms of systemic inflammatory response syndrome [27], upper gastrointestinal bleeding, hepatocellular carcinoma fulfilling Milan criteria [28] and/or portal thrombosis, previous liver transplantation, transjugular intrahepatic portosystemic shunt, alcoholic hepatitis, age older than 80 or younger than 18, etiology other than alcohol intake or HCV infection and refusal to participate in the study. The ethics committees (Comité Ético de Investigación Clínica del Hospital General de Alicante and Comité de Bioética de la Universidad de Murcia) approved the study protocol and all patients gave informed consent to be included in this study. A convenience sample of 61 consecutive patients who fulfilled all inclusion/exclusion criteria and who signed the informed consent were included in the study.

\section{Blood and ascites samples}

Peripheral blood and ascites were collected from patients with cirrhosis requiring a large-volume paracentesis at admission.

Blood was obtained for routine haematological, biochemical and coagulation studies. Simultaneously, a large-volume paracentesis was performed on all patients at admission in aseptic conditions following the usual procedures to obtain ascites [29]. All patients received intravenous albumin after paracentesis ( $8 \mathrm{~g} / \mathrm{l}$ of ascites) as routine protocol, if the volume of ascites evacuated was greater than 5 liters. Samples for routine biochemical study and PMN counts were obtained. Total protein, albumin, leukocyte and PMN counts were performed in all ascites specimens. Both blood and ascites were inoculated at bedside in aerobic and anaerobic blood culture bottles, $10 \mathrm{ml}$ each [30].

\section{Flow cytometry analysis}

Ascites samples were centrifuged at $500 \times \mathrm{G}$, the supernatant was collected for cytokine detection and cells were collected and washed in PBS, and then resuspended in DMEM (GIBCO Invitrogen, Paisley, UK). Cells from ascites were stained with monoclonal antibodies and analyzed by flow cytometry to determine cell types. Antibodies were mouse anti-human CD14-FITC (eBioscience, San Diego, CA), CD3-FITC, CD19-PE Cy5, CD14-PE and CD16-PE Cy5 (BD-Pharmingen, San Diego, CA). The mouse IgG1-PE, mouse IgG1-FITC, mouse IgG1-PE Cy5, mouse IgG2b-FITC and mouse IgG2b-PE antibodies used as isotype controls were from BD-Pharmingen. In brief, $0.3 \times 10^{6}$ cells in a volume of $100 \mu \mathrm{l}$ were stained with $5 \mu \mathrm{l}$ of the corresponding monoclonal antibodies and incubated in the dark on ice fixed in solution of lysis (Becton Dickinson, San José, 
CA) and then washed, resuspended in PBS and kept at $4^{\circ} \mathrm{C}$ in the dark until data acquisition.

Flow cytometry analyses were performed on threecolor fluorescence Epics XL (Beckman Coulter) using Cytomics RXP analysis software. 50,000-100,000 gated events were acquired and analyzed. Leukocytes were gated based on FCS vs. SSC (Forward vs. Side Scatter) on a lineal scale. Then, leukocyte subpopulations were gated on the base of morphology and $\mathrm{CD} 14^{+}$expression for peritoneal M-DM, $\mathrm{CD}^{+}$expression for $\mathrm{T}$ lymphocytes, CD $19^{+}$expression for B lymphocytes and $\mathrm{CD}^{-}$ CD14 ${ }^{-} \mathrm{CD} 19^{-} \mathrm{CD} 16^{+}$expression for NK cells.

\section{Isolation and stimulation of M-DM}

Cells were then seeded for panning at a ratio of $0.2 \times$ $10^{6} \mathrm{M}$-DM/well in 96-well plates for ELISA, or 1-2 $\times$ $10^{6} \mathrm{M}$-DM/well in 6-well plates for immunoblotting, according to the percentage of $\mathrm{CD} 14^{+}$cells determined by flow cytometry. After an overnight incubation at $37^{\circ} \mathrm{C}$ in DMEM containing 10\% fetal bovine serum and $1 \%$ penicillin/streptomycin (complete culture medium), cells were washed with complete culture medium to eliminate non-adhered cells, including lymphocytes. The purity of the M-DM in cell culture was more than $95 \%$. Then M-DM were maintained for 3 hours in serum starvation conditions (2\% FBS) and treated for 15 minutes with LPS (E. coli serotype 0111.B4, Sigma Aldrich Co, Saint Luis, Missouri, USA) for immunoblotting analysis of cell lysates, or directly with $0.1 \mu \mathrm{g} / \mathrm{ml}$ LPS, heat-killed C. albicans SC5314 strain at ratio $1 / 5$ cell/yeasts and synthetic phosphorothioate oligodeoxynucleotides (ODN) $(1 \mu \mathrm{g} / \mathrm{ml})$ presenting the following sequence 5' TGA CTG TGA ACG TTC GAG ATG A 3' (TriLink BioTechnologies, San Diego, CA) for ELISA. After 24 hours of incubation, cell culture supernatants were collected for cytokine detection.

\section{ELISA}

Ascitic fluid and cell culture supernatants were assayed by ELISA kits for TNF- $\alpha$, IL-1 $\beta$, IL-6, IL-10 and IL-12. The assay was performed in triplicate following the manufacturer's instructions ( $\odot R \& D$ Systems Inc., Minneapolis, USA). The absorbance in each well was measured with a microplate reader at $450 \mathrm{~nm}$ and corrected at $570 \mathrm{~nm}$.

\section{Cellular lysates and immunoblotting}

Protein extracts were obtained and treated as described elsewhere [31]. Primary antibodies against phosphorylated ERK1/2 (Thr202/Tyr204) and $\beta$-actin (Sigma Aldrich Co, Saint Luis, Missouri, USA) were used before incubation with the corresponding HRP-conjugated secondary antibody. The activity of membrane-bound peroxidase was detected by using an enhanced chemiluminescent detection method (Enhanced ChemiLuminescence system, @Amersham Pharmacia Biotech; Piscataway, NJ, USA). Protein bands were quantified by densitometry using Scion Image software and expressed relative to $\beta$-actin.

\section{Statistical analysis}

Categorical variables are reported as frequency or percentages. Continuous variables are reported as median and interquartile range (IQR) and are graphically represented as box diagrams, where the top and bottom of the box are the $25^{\text {th }}$ and $75^{\text {th }}$ percentile (the lower and upper quartiles, respectively), and the band inside the box is the $50^{\text {th }}$ percentile (the median), the end of the whiskers represent the lowest datum still within 1.5 IQR of the lower quartile, and the highest datum still within 1.5 IQR of the upper quartile. The observations considered as outliers are shown as circles outside the boxes. Statistic differences were analyzed using the MannWhitney $U$ test or Wilcoxon signed-rank test. All reported $\mathrm{P}$ values are two-sided, and $\mathrm{P}$ values lower than 0.05 were considered to indicate statistical significance. All calculations were performed using the SPSS 15.0 software (Chicago, IL, USA).

Reporting of the study conforms to STROBE and EQUATOR guidelines [32].

\section{Abbreviations}

ALD: Alcoholic liver disease; HCV: Hepatitis C Virus; ALC-C: Alcohol induced cirrhosis; HCV-C: HCV Induced Cirrhosis; M-DM: Monocyte-derived macrophages; PAMP: Pathogen-associated molecular pattern; PRR: PAMPRecognition receptor; PMN: PolyMorphoNuclear;

ODN: OligoDeoxyNucleotide; SBP: Spontaneous bacterial peritonitis.

\section{Competing interests}

The authors declare that they have no competing interest.

\section{Authors' contributions}

ATA participated in the isolation and stimulation of M-DM, ELISA, immunoblotting and statistical analysis. MME participated in the isolation and stimulation of M-DM, ELISA, statistical analysis, the design of the study and drafted the manuscript, AJRA participated in the isolation and stimulation of M-DM and immunoblotting, THC carried out the flow cytometry analysis, CMP participated in the acquisition of blood and ascites samples, clinical and analytical data and informed consent from patients. MML participated in the design of the study. JS participated in the design of the study. RF participated in the acquisition of blood and ascites samples, clinical and analytical data, informed consent from patients and in the design of the study. PGP participated in the design and coordination of the study and helped to draft the manuscript. All authors read and approved the final manuscript.

\section{Acknowledgements}

This study was funded by grant 11926/PI/09 from the Fundación Séneca, Comunidad Autónoma de la Región de Murcia, Spain. Ana Tapia-Abellán was supported by the Fundación Séneca (12302/FPI/09), Comunidad Autónoma de la Región de Murcia, Spain. We thank Antonio Maurandi López, from the Servicio de Estadística del SAI (Servicio de Apoyo a la Investigación),

University of Murcia for his help with the statistics.

\section{Author details}

'Departamento de Bioquímica, Biología Molecular (B) e Inmunología Facultad de Medicina, Universidad de Murcia, Murcia 30100, Spain. ²Unidad de Trasplante Hepático, Servicio de Aparato Digestivo, Hospital Universitario Virgen de la Arrixaca, Murcia, Spain. ${ }^{3}$ Unidad Hepática, Hospital General Universitario, Alicante, Spain. ${ }^{4}$ CIBERehd, Instituto de Salud Carlos III, Madrid, Spain. 
Received: 2 April 2012 Accepted: 24 July 2012

Published: 6 August 2012

\section{References}

1. Mas VR, Fassnacht R, Archer K, Maluf D: Molecular mechanisms involved in the interaction effects of alcohol and hepatitis $C$ virus in liver cirrhosis. Mol Med 2010, 16:287-297.

2. Chen CM, Yoon YH, Yi HY, Lucas DL: Alcohol and hepatitis C mortality among males and females in the United States: a life table analysis. Alcohol Clin Exp Res 2007, 31:285-292.

3. Wise M, Bialek S, Finelli L, Bell BP, Sorvillo F: Changing trends in hepatitis C-related mortality in the United States, 1995-2004. Hepatology 2008, 47:1128-1135.

4. Uchimura Y, Sata M, Kage M, Abe H, Tanikawa K: A histopathological study of alcoholics with chronic HCV infection: comparison with chronic hepatitis C and alcoholic liver disease. Liver 1995, 15:300-306.

5. Lederer SL, Walters KA, Proll S, Paeper B, Robinzon S, Boix L, et al: Distinct cellular responses differentiating alcohol- and hepatitis $C$ virus-induced liver cirrhosis. Virol J 2006, 3:98.

6. Miller AM, Horiguchi N, Jeong WI, Radaeva S, Gao B: Molecular mechanisms of alcoholic liver disease: innate immunity and cytokines. Alcohol Clin Exp Res 2011, 35:787-793.

7. McClain CJ, Song Z, Barve SS, Hill DB, Deaciuc I: Recent advances in alcoholic liver disease. IV. Dysregulated cytokine metabolism in alcoholic liver disease. Am J Physiol Gastrointest Liver Physiol 2004, 287:G497-G502.

8. Heller J, Sogni P, Barriere E, Tazi KA, Chauvelot-Moachon L, Guimont MC, et al: Effects of lipopolysaccharide on TNF-alpha production, hepatic NOS2 activity, and hepatic toxicity in rats with cirrhosis. $J$ Hepatol 2000, 33:376-381.

9. Frances R, Munoz C, Zapater P, Uceda F, Gascon I, Pascual S, et al: Bacterial DNA activates cell mediated immune response and nitric oxide overproduction in peritoneal macrophages from patients with cirrhosis and ascites. Gut 2004, 53:860-864.

10. Ruiz-Alcaraz AJ, Martinez-Esparza M, Cano R, Hernandez-Caselles T, Recarti C, Llanos $L$, et al: Peritoneal macrophage priming in cirrhosis is related to ERK phosphorylation and IL-6 secretion. Eur J Clin Invest 2011, 41:8-15.

11. Ferri S, Lalanne C, Lanzoni G, Bassi M, Asioli S, Cipriano V, et al: Redistribution of regulatory T-cells across the evolving stages of chronic hepatitis C. Dig Liver Dis 2011, 43:807-813.

12. Li WY, Jiang YF, Jin QL, Zhang H, Feng XW, Niu JQ: Immunologic characterization of posthepatitis cirrhosis caused by HBV and HCV infection. J Biomed Biotechnol 2010, 201:138237.

13. Lemmers A, Moreno C, Gustot T, Marechal R, Degre D, Demetter P, et al: The interleukin-17 pathway is involved in human alcoholic liver disease. Hepatology 2009, 49:646-657.

14. Bonacini M, Govindarajan S, Kohla M, Lai MM, Lindsay KL: Intrahepatic lymphocyte phenotypes in hepatitis C virus infection: a comparison between cirrhotic and non-cirrhotic livers. Minerva Gastroenterol Dietol 2007, 53:1-7.

15. Ribera J, Pauta M, Melgar-Lesmes P, Tugues S, Fernandez-Varo G, Held KF, et al: Increased nitric oxide production in lymphatic endothelial cells causes impairment of lymphatic drainage in cirrhotic rats. Gut 2012, in press. doi: gutjnl-2011-300703.

16. Zimmermann HW, Seidler S, Nattermann J, Gassler N, Hellerbrand C, Zernecke $\mathrm{A}$, et al: Functional contribution of elevated circulating and hepatic non-classical CD14CD16 monocytes to inflammation and human liver fibrosis. PLoS One 2010, 5:e11049.

17. Zimmermann HW, Seidler S, Gassler N, Nattermann J, Luedde T, Trautwein C, et al: Interleukin-8 is activated in patients with chronic liver diseases and associated with hepatic macrophage accumulation in human liver fibrosis. PLoS One 2011, 6:e21381.

18. Delhem N, Cottrez F, Carpentier A, Miroux C, Morales O, Francois V, et al: Role of the regulatory $\mathrm{T}$ lymphocytes in hepatitis $\mathrm{C}$ fibrosis progression. Bull Cancer 2008, 95:1029-1038.

19. Osna NA: Hepatitis $C$ virus and ethanol alter antigen presentation in liver cells. World J Gastroenterol 2009, 15:1201-1208.

20. Sreenarasimhaiah J, Jaramillo A, Crippin J, Lisker-Melman M, Chapman WC, Mohanakumar T: Lack of optimal T-cell reactivity against the hepatitis $C$ virus is associated with the development of fibrosis/cirrhosis during chronic hepatitis. Hum Immunol 2003, 64:224-230.
21. Lin CY, Tsai IF, Ho YP, Huang CT, Lin YC, Lin CJ, et al: Endotoxemia contributes to the immune paralysis in patients with cirrhosis. J Hepatol 2007, 46:816-826.

22. Dolganiuc A, Garcia C, Kodys K, Szabo G: Distinct Toll-like receptor expression in monocytes and T cells in chronic HCV infection. World J Gastroenterol 2006, 12:1198-1204.

23. Mozer-Lisewska I, Sluzewski W, Kaczmarek M, Jenek R, Szczepanski M, Figlerowicz $\mathrm{M}$, et al: Tissue localization of Toll-like receptors in biopsy specimens of liver from children infected with hepatitis $C$ virus. Scand I Immunol 2005, 62:407-412.

24. Seki E, Brenner DA: Toll-like receptors and adaptor molecules in liver disease: update. Hepatology 2008, 48:322-335.

25. Such J, Runyon BA: Spontaneous bacterial peritonitis. Clin Infect Dis 1998, 27:669-674.

26. Albillos A, Cuervas-Mons V, Millan I, Canton T, Montes J, Barrios C, et al: Ascitic fluid polymorphonuclear cell count and serum to ascites albumin gradient in the diagnosis of bacterial peritonitis. Gastroenterology 1990, 98:134-140.

27. Rangel-Frausto MS, Pittet D, Costigan M, Hwang T, Davis CS, Wenzel RP: The natural history of the systemic inflammatory response syndrome (SIRS). A prospective study. JAMA 1995, 273:117-123.

28. Mazzaferro V, Regalia E, Doci R, Andreola S, Pulvirenti A, Bozzetti F, et al: Liver transplantation for the treatment of small hepatocellular carcinomas in patients with cirrhosis. N Engl J Med 1996, 334:693-699.

29. Runyon BA: Paracentesis of ascitic fluid. A safe procedure. Arch Intern Med 1986, 146:2259-2261.

30. Runyon BA, Canawati HN, Akriviadis EA: Optimization of ascitic fluid culture technique. Gastroenterology 1988, 95:1351-1355.

31. Zapater P, Cano R, Llanos L, Ruiz-Alcaraz AJ, Pascual S, Barquero C, et al: Norfloxacin modulates the inflammatory response and directly affects neutrophils in patients with decompensated cirrhosis. Gastroenterology 2009, 137:1669-1679.

32. Simera I, Moher D, Hoey J, Schulz KF, Altman DG: A catalogue of reporting guidelines for health research. Eur J Clin Invest 2010, 40:35-53.

doi:10.1186/1471-2172-13-42

Cite this article as: Tapia-Abellán et al:: The peritoneal macrophage inflammatory profile in cirrhosis depends on the alcoholic or hepatitis C viral etiology and is related to ERK phosphorylation. BMC Immunology 2012 13:42.

\section{Submit your next manuscript to BioMed Central and take full advantage of:}

- Convenient online submission

- Thorough peer review

- No space constraints or color figure charges

- Immediate publication on acceptance

- Inclusion in PubMed, CAS, Scopus and Google Scholar

- Research which is freely available for redistribution 